\title{
Aнenponempoвский zocyнusepcumem
}

\section{Оптимизачия "интервапьнык» квадратурных формул Aля kласеов $\mathrm{H}^{\omega_{+} \omega_{-}}$}

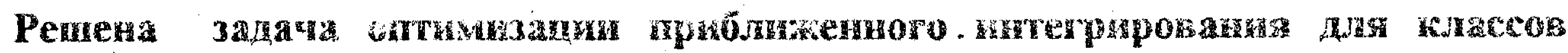

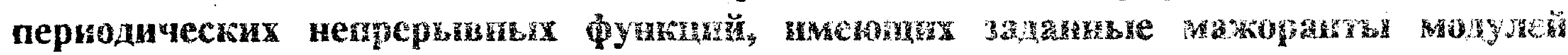

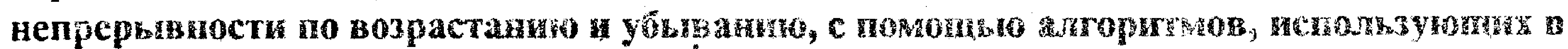

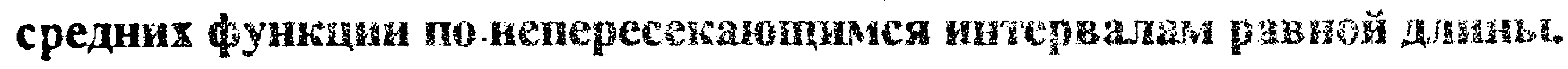

Задача оптимизации кеадратурньг фориул на различицх классах функций одной и многих переменнви восходит к работам А.H. Солмоторова и С.М. Никольского конца 40-x - начала 50-x roдов. Большое количество работ было посвящено оптимизации для различньк классов функций формул приближенного вычисления интегралов, использующих значения функции в $\mathrm{n}$ точках (узлах). Киложение многих полученньх в этом направлении результатов можно найти в монографии [5]. Наряду с этим, в ряде работ (см., напр., [1],[4],[6] - [8] ) рассматривались заадачи оптимизации так называемьх интервальньх квадратурньх формул, в которых вместо значений функции в узлах использовались ее средние значения по некоторым интервалам в области определения ( определенном смысле, приближенное интетрирование функций с помошью формул второго типа является более естественньм, чем приближенное интегрирование с помощью формул первого типа: ках правило; результат измерения - это усреднение измеряемой функиии по некоторому интервалу). .

В работе [6] решіена задача о наилучших квадратурных формулах для функций классов Липшица с показателем 1 и константой $M$, определениых на отрезке. Цель ганной работы - рассмотреть задачу оттимзацй "интервальиьх" квадратурных формул на классах непрерьвных периодическа функций, ммеющи заданные выпукле вверх мажоранты модуля непрерызности по возрастанио и убыванию (см. определения ниже). Пуст С - пространство $2 \pi$-гериодихеских веществениьх непрерывньг функий, заданных на числовой оси. Дпл $\mathrm{f} \in \mathrm{C}$, положим

$$
\begin{aligned}
\omega(f, t) & =\sup _{\left|x^{\prime}-x^{\prime \prime}\right| \leq t} \mid f\left(x^{\prime}\right)-f\left(x^{\prime \prime}\right),, t \geq 0, \\
\omega_{ \pm}(f, t)= & \sup _{\substack{\left|x^{\prime}-x^{\prime \prime}\right| \leq t \\
t x^{\prime} \geq x^{\prime}}}\left(f\left(x^{\prime}\right)-f\left(x^{\prime \prime}\right)\right), t \geq 0
\end{aligned}
$$

Функиия (1) - модуль нетрерцвности функиии $f$ Функико $\omega_{+}(f, t)$ пазовем

С Бородачез С.B., 1998 
модулем непрерывности функции f по возрастанию, a $\omega_{-}(f, t)$ модулем кетрернивности $f$ по убыванию.

Пусть $\omega_{+}, \omega_{-}-$заданные модули непрерывности. Через $\mathrm{H}^{\omega_{+} ; \omega_{-}}$ обозиачим класс функций $f \in C$, дия которьх $\omega_{ \pm}(f, t) \leq \omega_{ \pm}(t), \mathfrak{t} \geq 0$. Мы будем рассматривать задаяу оптимизации «интервальньх» квадратурных формул на классе $\mathrm{H}^{\omega_{*} ; \omega_{-}}$, где $\omega_{+}(\mathrm{t}), \omega_{-}(\mathrm{t})$ - выпуклые вверх модули непрерывности, в следующей постановке.

Пусть заданы числа $h>0, n \in N\left(n<\frac{\pi}{h}\right)$. Через $D_{h, n}$ обозначим множество всевозможных функционалов вида

$$
S(f)=\varphi\left(\frac{1}{2 h} \int_{x_{1}-h}^{x_{1}+h} f(t) d t, \ldots, \frac{1}{2 h} \int_{x_{2}-h}^{x_{n}+h} f(t) d t\right),
$$

где $0 \leq x_{1}<\ldots<x_{n}<2 \pi, x_{k}-x_{k-1}>2 h, k=2, \ldots, n, x_{1}+2 \pi-x_{n}>2 h ;$ функция $\varphi: \mathbf{R}^{\mathbf{n}} \rightarrow \mathbf{R}-$ произвольная.

Положим

$$
\begin{gathered}
R(f ; S)=\int_{0}^{2 \pi} f(t) d t-S(f), \\
R\left(H^{\omega_{+} ; \omega_{-}} ; S\right)=\sup _{f \in H^{m_{+}} ;} \mid R(f ; S), \\
R_{h, n}\left(H^{\omega_{+} ; \omega_{-}}\right)=\inf _{S \in D_{b .}} R\left(\mathrm{H}^{\omega_{+} ; \omega_{-}} ; S\right) .
\end{gathered}
$$

Требуется найти величину (3) и указать оптимальную квадратурную формулу из множества $\mathrm{D}_{\mathrm{h}, \mathrm{n}}$ (то есть формулу реализующую точную нижнюю грань в правой части равенства (3)).

Решение задачи оттимизации приближенного интегрирования функций класса $\mathrm{H}^{\omega_{+} ; \omega_{-}}$в такой постановке дается следующей теоремой.

Теорема . Пусть заданы.числа $\mathrm{h}>0, \mathrm{n} \in \mathrm{N}\left(\mathrm{n}<\frac{\pi}{\mathrm{h}}\right)$, и выпуклые вверх модули непрерывности $\omega_{+}(t)$ и $\omega_{-}(t)$. Среди всевозможных квадратурных формул из множества $\mathrm{D}_{\mathrm{h}, \mathrm{n}}$ оттимальной на классе $\mathrm{H}^{\omega_{+}}$; $\omega_{-}$является формула

где $\tau \in\left[0, \frac{2 \pi}{n}\right)$. При этом

$$
S^{*}(f)=\frac{2 \pi}{n} \sum_{k=1}^{n} \frac{1}{2 b} \int_{t+\frac{2 x}{n}(k-1)-b}^{i+\frac{2 \pi}{n}(k-1)+b} f(t) d t
$$

$$
R_{h, n}\left(H^{\omega_{+} ; \omega_{-}}\right)=n\left(1-\frac{h n}{\pi}\right) \int_{0}^{\frac{2 \pi}{n}} \min \left\{\omega_{+}(t), \omega_{-}\left(\frac{2 \pi}{n}-t\right)\right) d t .
$$

Доказательство. Пусть $\mathrm{h}>0, \mathrm{n}$ - натуральное число $\left(\mathrm{n}<\frac{\pi}{\mathrm{h}}\right)$, $\omega_{+}(t) \omega_{-}(t)$ - выпуклые вверх модули непрерывности. Если один из модулей непрерывности тождественно равен 0 , то утверждение теоремы . очевидно, 
т.к. $\mathrm{H}^{\Phi_{+} ; \omega_{-}}$будет состоять лишь из констант. Поэтому будем считать, что $\omega_{+}(t)$ и $\omega_{-}(t)$ не являются тождественно равными 0.

Покажем вначале, что

$$
\mathrm{R}\left(\mathrm{H}^{\omega_{+} ; \omega_{-}} ; \mathrm{S}^{*}\right) \leq \mathrm{n}\left(1-\frac{\mathrm{hn}}{\pi}\right) \int_{0}^{\frac{2 \pi}{\mathrm{n}}} \min \left\{\omega_{+}(\mathrm{t}), \omega_{-}\left(\frac{2 \pi}{\mathrm{n}}-\mathrm{t}\right)\right\} \mathrm{t}
$$

Не нарушая общности, можно считать, что $\tau=0$. Для любой функщии $f \in \mathrm{H}^{\omega_{+} ; \omega_{-}}$имеем

$R\left(f ; S^{*}\right)=\int_{0}^{2 \pi} f(t) d t-\sum_{k=12 \pi(k-1)}^{n} \frac{\frac{2 \pi(k-1)}{n}}{n} \frac{\pi}{h n} f(t) d t=\int_{0}^{2 \pi} f(t) \psi(t) d t$

где $\psi: R \rightarrow R-\frac{2 \pi}{\mathbf{n}}$ - периодическая функция, задаваемая на периоде $\left[-h, \frac{2 \pi}{n}-h\right)$ следующим образом :

$$
\psi(t)=\left\{\begin{array}{l}
1-\frac{\pi}{h n}, t \in[-h, h] \\
1, \quad t \in\left(h, \frac{2 \pi}{n}-h\right)
\end{array}\right.
$$

Заметим, что

$$
R\left(H^{\omega_{+} ; \omega_{-}} ; S^{*}\right)=\sup _{f \in H^{\omega_{+} ; \alpha_{-}}} R\left(f ; S^{*}\right)
$$

В самом деле, для любой функции $\mathrm{f} \in \mathrm{H}^{\omega_{+} ; \omega_{-}}, \mathrm{g}(\mathrm{t})=-\mathrm{f}(-\mathrm{t})$ также принадлежит $\mathrm{H}^{\omega_{+} ; \omega_{-}}$. Пользуясь четностью функции $\psi$, будем иметь

$$
\mathrm{R}\left(\mathrm{g} ; \mathrm{S}^{*}\right)=\int_{0}^{2 \pi} \mathrm{g}(\mathrm{t}) \psi(\mathrm{t}) \mathrm{dt}=-\int_{0}^{2 \pi} \mathrm{f}(\mathrm{t}) \psi(\mathrm{t}) \mathrm{dt}=-\mathrm{R}\left(\mathrm{f} ; \mathrm{S}^{*}\right)
$$

тогда

$$
\sup _{f \in H^{*+*}} R\left(f ; S^{*}\right)=-\inf _{f \in H^{*+*}} R\left(f ; S^{*}\right)
$$

откуда и следует (5). Заметим также, что

$$
R\left(\mathrm{H}^{\omega_{+} ; \omega_{-}} ; \mathrm{S}^{*}\right)=\sup _{\mathrm{f} \in \mathrm{Y}} \mathrm{R}\left(\mathrm{f} ; \mathrm{S}^{*}\right)
$$

где $\mathrm{Y}$ - множество $\frac{2 \pi}{\mathrm{n}}$ - периодических функций класса $\mathrm{H}^{\omega_{+} ; \omega_{-}}$, так как для гобой функции $f \in H^{\omega_{+} ; \omega_{-}}$функция $\gamma: R \rightarrow R$, равная

$$
\gamma(t)=\frac{1}{n} \sum_{k=0}^{n-1} f\left(t+\frac{2 \pi k}{n}\right)
$$




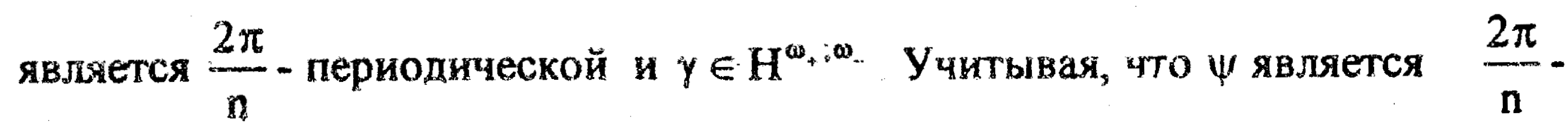
периодической функцией, получим

$$
\begin{aligned}
R\left(\gamma ; S^{*}\right) & =\int_{0}^{2 \pi} \gamma(t) \psi(t) d t=n \int_{0}^{\frac{2 \pi}{n}}\left(\sum_{k=0}^{n-1} \frac{1}{n} f\left(t+\frac{2 \pi k}{n}\right)\right) \psi(t) d t=\sum_{k=0}^{n-1} \int_{0}^{n-1} f\left(t+\frac{2 \pi k}{n}\right) \psi(t) d t= \\
& =\sum_{k=0}^{n-1} \frac{2 \pi(k+1)}{n} f(t) \psi\left(t-\frac{2 \pi k}{n}\right) d t=\sum_{k=0}^{n-1} \frac{2 \pi(k+1)}{\int_{\frac{2 \pi k}{n}}^{n} f(t)} \psi(t) d t=\int_{0}^{2 \pi} f(t) \psi(t) d t=R\left(f ; S^{*}\right)
\end{aligned}
$$

откуда и следует (6).

Пусть у - решение уравнения $\omega_{+}(x)=\omega_{-}\left(\frac{2 \pi}{n}-x\right)$ на интервале $\left(0, \frac{2 \pi}{\mathrm{n}}\right)$. Положим $\delta=\frac{\mathrm{n}}{2 \pi} \mathrm{y}, \mu_{0}=-\mathrm{h}-2(1-\delta)\left(\frac{\pi}{\mathrm{n}}-\mathrm{h}\right), \mu_{1}=-\mathrm{h}+2(1-\delta) \mathrm{h}$. Легко видеть, что $-\frac{2 \pi}{\mathrm{n}}+\mathrm{h}<\mu_{0}<-\mathrm{h}<\mu_{1}<\mathrm{h}<\mu_{0}+\frac{2 \pi}{\mathrm{n}}$. Для любой функции $\gamma \in \mathrm{Y}$

$\mathrm{R}\left(\gamma ; \mathrm{S}^{*}\right)=\int_{0}^{2 \pi} \gamma(\mathrm{t}) \psi(\mathrm{t}) \mathrm{dt}=\mathrm{n} \int_{\mu_{0}}^{\frac{2 \pi}{n}+\mu_{0}} \gamma(\mathrm{t}) \psi(\mathrm{t}) \mathrm{dt}=\mathrm{n}\left(\int_{\mu_{0}}^{\mu_{1}} \gamma(\mathrm{t}) \psi(\mathrm{t}) \mathrm{dt}+\int_{\mu_{0}}^{\frac{2 \pi}{n}+\mu_{0}} \gamma(\mathrm{t}) \psi(\mathrm{t}) \mathrm{dt}\right) \leq$

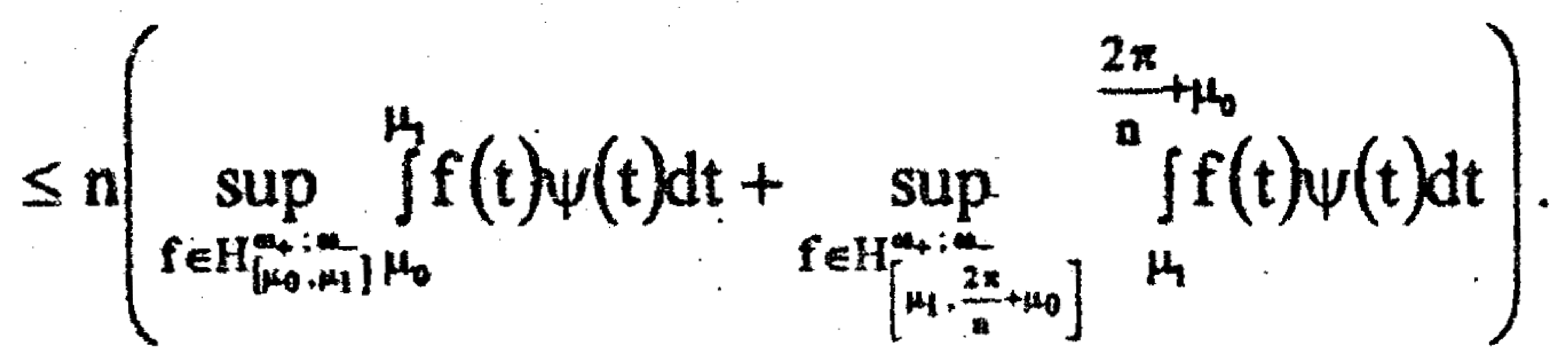

- Дия вычисления точньх верхних граней воспользуемся следующим вариантом леммы Корнейчука - Стечкина [3, лемма 7.4.1], который получен B [2].

Лемма . Пусть суммируемая на $(\alpha, \beta)$ функция $\psi_{1}(t)$ удовлетворяет следующим условиям:

1) функция $\Psi(\mathrm{x})=\int_{\alpha}^{\mathrm{x}} \psi_{1}(\mathrm{t}) \mathrm{dt} ; \alpha \leq \mathrm{x} \leq \beta$, строго монотонна на $\left(\alpha, \alpha^{\prime}\right)$ и $\left(\beta^{\prime}, \beta\right), \alpha<\alpha^{\prime} \leq \beta^{\prime}<\beta, \Psi(\beta)=0, \max _{\alpha \leq x \leq \beta}|\Psi(x)|=|\Psi(t)|, \alpha^{\prime} \leq t \leq \beta^{\prime} ;$

2) на промежутках $\left(\alpha, \alpha^{\prime}\right)$ и $\left(\beta^{\prime}, \beta\right)$ функция $\psi_{1}(t)$ обращается в нуль разве чтө на множестве меры нуль. Пусть, далее, $\omega_{+}(\mathrm{t}), \omega_{-}(\mathrm{t})$ - выпуклые вверх модули непрерывности. Тогда, если $\pm \psi_{1}(t) \leq 0$ для $\mathrm{x} \in\left(\alpha, \alpha^{\prime}\right)$, то

$$
\sup _{f \in H_{\left.[\alpha, \beta]^{+}\right]}} \int_{\alpha}^{\beta} f(t) \psi_{1}(t) d t=\int_{0}^{\beta-\alpha} P_{[\alpha, \beta]}(|\Psi|, t) \omega_{ \pm}^{\prime}(t) d t
$$


где $P_{[\alpha, \beta]}(\rho, t)$ - убывающая перестановка $[3$, с. 130] фзнкщии $\rho \geq 0$, определенной на $(\alpha, \beta) ; \quad \mathrm{H}_{[\alpha, \beta]}^{\omega_{+} ; \omega_{-}}-$кјасс неирерывных функций, определенных на отрезке $[\alpha, \beta]$ с заданными мажорантами модуля непрерывности по возрастанию $\left(\omega_{+}\right)$и убыванию $\left(\omega_{-}\right)$.

В рассматриваемой задаче сужения функции $\psi$ на отрезок $\left[\mu_{0}, \mu_{1}\right]$ и на отрезок $\left[\mu_{1}, \mu_{0}+\frac{2 \pi}{n}\right]$ удовлетворяют условиям леммы. ", $\omega_{+}, \omega_{-}-$ выпуклые вверх модули непрерывности, поэтому

$$
\left.\left.\mathrm{R}\left(\gamma ; \mathrm{S}^{*}\right) \leq \mathrm{n}\left(\int_{0}^{\mu_{-}-\mu_{0}} \mathrm{P}_{\left[\mu_{0}, \mu_{1}\right]}\right]\left(\Psi_{1} \mid, t\right)\right)_{\omega_{-}^{\prime}}(\mathrm{t}) \mathrm{dt}+\int_{0}^{\mu_{0}+\frac{2 \pi}{n} \mu_{1}} \mathrm{P}_{\left[\mu_{1}, \mu_{0}+\frac{2 \pi}{n}\right]}\left(\left|\Psi_{2}\right|, t\right) \omega_{+}^{\prime}(\mathrm{t}) \mathrm{dt}\right)
$$

где $\Psi_{1}(x)=\int_{\mu_{0}}^{x} \psi(t) d t, x \in\left[\mu_{0}, \mu_{1}\right], \Psi_{2}(x)=\int_{\mu_{1}}^{x} \psi(t) d t, x \in\left[\mu_{1}, \mu_{0}+\frac{2 \pi}{n}\right]$.

Вычислив убывающие перестановки $\left|\Psi_{1}\right|,\left|\Psi_{2}\right|$, получим

$$
\begin{gathered}
\mathrm{R}\left(\gamma ; \mathrm{S}^{*}\right) \leq \mathrm{n}\left(\frac{\mathrm{nh}}{\pi}-1\right)\left(\int_{0}^{\mu_{1}-\mu_{2}}\left(\mathrm{t}-\left(\mu_{1}-\mu_{0}\right)\right) \omega_{-}^{\prime}(\mathrm{t}) \mathrm{dt}+\right. \\
\left.+\int_{0}^{\mu_{0}+\frac{2 \pi}{n} \mu_{1}}\left(\mathrm{t}-\left(\mu_{0}+\frac{2 \pi}{\mathrm{n}}-\mu_{1}\right)\right) \omega_{+}^{\prime}(\mathrm{t}) \mathrm{dt}\right)
\end{gathered}
$$

ннтегрируя по частям и учитывая, что $\mu_{1}-\mu_{0}=\frac{2 \pi}{\mathrm{n}}-\mathrm{y}, \mu_{0}+\frac{2 \pi}{\mathrm{n}}-\mu_{1}=\mathrm{y}$, будем иметь

Таким образом,

$$
\begin{gathered}
\mathrm{R}\left(\gamma ; \mathrm{S}^{*}\right) \leq \mathrm{n}\left(1-\frac{\mathrm{nh}}{\pi}\right)\left(\int_{0}^{\frac{2 \pi}{\mathrm{n}} \mathrm{y}} \omega_{-}(\mathrm{t}) \mathrm{dt}+\int_{0}^{\mathrm{y}} \omega_{+}(\mathrm{t}) \mathrm{dt}\right)= \\
\quad=\mathrm{n}\left(1-\frac{\mathrm{hn}}{\pi}\right) \int_{0}^{\frac{2 \pi}{n}} \min \left\{\omega_{+}(\mathrm{t}), \omega_{-}\left(\frac{2 \pi}{\mathrm{n}}-\mathrm{t}\right) \mathrm{dt} .\right.
\end{gathered}
$$

$$
R\left(H^{\omega_{*} ; \omega_{-}} ; S^{*}\right)=\sup _{f \in Y} R\left(f ; S^{*}\right) \leq n\left(1-\frac{h n}{\pi}\right) \int_{0}^{\frac{2 \pi}{n}} \min \left\{\left(\omega_{+}(t), \omega_{-}\left(\frac{2 \pi}{n}-t\right)\right) d t .\right.
$$

Покажем, что погрешность на классе $\mathrm{H}^{\omega_{+} ; \omega_{-}}$любого цругого әтгоритма из множества $\mathrm{D}_{\mathrm{h}, \mathrm{n}}$ не меньше величины, стоящей в правой части керавенства (7).

Пусть задан произяольный алгоритм $\mathrm{S} \in \mathrm{D}_{\mathrm{h}, \mathrm{n}}$. Построим две функции 
$\mathrm{g}_{1}, \mathrm{~g}_{2} \in \mathrm{H}^{\omega_{+} ; \omega_{-}}$следующим образом.

Пусть $\mathrm{g}_{0}: \mathrm{R} \rightarrow \mathbf{R}$ - следующая функция :

$$
g_{0}(t)=\left\{\begin{array}{l}
\left(1-\frac{h n}{\pi}\right) \omega_{-}\left(\frac{-h-t}{\pi-h n} \pi\right), t \in\left[\mu_{0},-h\right), \\
-\frac{h n}{\pi} \omega_{-}\left(\frac{t+h}{h n} \pi\right), t \in\left[-h, \mu_{1}\right), \\
-\frac{h n}{\pi} \omega_{+}\left(\frac{\pi}{h n}(h-t)\right), t \in\left[\mu_{1}, h\right), \\
\left(1-\frac{h n}{\pi}\right) \omega_{+}\left(\frac{t-h}{\pi-h n} \pi\right), t \in\left[h, \mu_{0}+\frac{2 \pi}{n}\right) \\
\left(1-\frac{h n}{\pi}\right) \omega_{-}\left(\frac{-h-\mu_{0}}{\pi-h n} \pi\right), t \in\left[\mu_{0}, \mu_{0}+\frac{2 \pi}{n}\right)
\end{array}\right.
$$

и пусть $\overline{\mathrm{g}}_{0}(\mathrm{t})=\mathrm{g}_{0}(\mathrm{t})-\frac{1}{2 \mathrm{~h}} \int_{-\mathrm{h}}^{\mathrm{h}} \mathrm{g}_{0}(\mathrm{v}) \mathrm{dv}$. Нетрудно проверить, что $\overline{\mathrm{g}}_{0}$ непрерывна на всей числовой прямсй и $\omega_{ \pm}\left(\bar{g}_{0}, t\right) \leq \omega_{ \pm}(t), t \geq 0$. Пусть, для удобства, $x_{n+r}=x_{1}+2 \pi$. Для $k=1, \ldots, n$ обозначим через $y_{k}$ решение уравнения

$$
\overline{\mathrm{g}}_{0}\left(\mathrm{t}-\mathrm{x}_{\mathrm{k}}\right)=\overline{\mathrm{g}}_{0}\left(\mathrm{t}-\mathrm{x}_{\mathrm{k}+1}\right)
$$

на отрезке $\left[x_{k}+h, x_{k+1}-h\right]$. (Оно существует, так как разность $\bar{g}_{0}\left(t-x_{k}\right)-\bar{g}_{0}\left(t-x_{k+1}\right)$ меняет знак на этом отрезке) Обозначим также $\mathrm{y}_{0}=\mathrm{y}_{\mathrm{n}}-2 \pi$. Пусть $\mathrm{g}_{1}-2 \pi-$ периодическая функция, определенная на периоде $\left(\mathrm{y}_{0}, \mathrm{y}_{\mathrm{n}}\right)$ следующим образом:

$$
g_{1}(t)=\bar{g}_{0}\left(t-x_{k}\right), t \in\left[y_{k-1}, y_{k}\right), k=1, \ldots, n \text {. }
$$

Для $\mathrm{k}=1, \ldots, \mathrm{n}$ обозначим через $\mathrm{z}_{\mathrm{k}}$ решение уравнекия $-\overline{\mathrm{g}}_{0}\left(\mathrm{x}_{\mathrm{k}}-\mathrm{t}\right)=-\overline{\mathrm{g}}_{0}\left(\mathrm{x}_{\mathrm{k}+1}-\mathrm{t}\right)$ на отрезке $\left[\mathrm{x}_{\mathrm{k}}+\mathrm{h}, \mathrm{x}_{\mathrm{k}+1}-\mathrm{h}\right]$ и положим $z_{0}=z_{n}-2 \pi$. Пусть $g_{2}-2 \pi-$ периодическая функция, определенная на периоде $\left[z_{0}, z_{n}\right)$ так:

$$
g_{2}(t)=-\bar{g}_{0}\left(x_{k}-t\right), t \in\left[z_{k-1}, z_{k}\right), \quad k=1 ; \ldots, n .
$$

Нетрудно убедиться, что $\mathrm{g}_{1}, \mathrm{~g}_{2} \in \mathrm{H}^{\infty_{+} ; \infty_{-}}$.

Тогда для выбранного алгоритма $S$

$$
\begin{aligned}
& R\left(H^{\omega_{*} ; \omega_{-}} ; S\right) \geq \max _{i=1,2}\left|R\left(g_{i} ; S\right) \geq \frac{1}{2} \sum_{i=1}^{2}\right| \int_{0}^{2 x} g_{i}(t) d t-\varphi\left(\frac{1}{2 h} \int_{x_{i}-h}^{x_{1}+h} g_{i}(t) d t, \ldots, \frac{1}{2 h} \int_{x_{i}-h}^{x_{n}+h} g_{i}(t) d t\right) \mid \geq \\
& \geq \frac{1}{2} \int_{0}^{2 \pi} g_{1}(t) d t-\varphi\left(\frac{1}{2 h} \int_{x_{1}-h}^{x_{1}+b} g_{1}(t) d t, \ldots, \frac{1}{2 h} \int_{x_{n}-h}^{x_{2}+h} g_{1}(t) d t\right)-\int_{0}^{2 \pi} g_{2}(t) d t+
\end{aligned}
$$


$\left.+\varphi\left(\frac{1}{2 \mathrm{~b}} \int_{\mathrm{x}_{1}-\mathrm{b}}^{\mathrm{x}_{1}+\mathrm{b}} \mathrm{g}_{2}(\mathrm{t}) \mathrm{dt}, \ldots, \frac{1}{2 \mathrm{~b}} \int_{\mathrm{x}_{\mathrm{n}}-\mathrm{b}}^{\mathrm{x}_{\mathrm{n}}+\mathrm{b}} \mathrm{g}_{2}(\mathrm{t}) \mathrm{dt}\right)\right)$.

Ta: как $\bar{g}_{0}(t) h \bar{g}_{0}(-t)$ на отрезке $[-\mathrm{h}, \mathrm{h}]$ в среднем равны нулю, то средние функций $\mathrm{g}_{1}$ и $\mathrm{g}_{2}$ по всем интервалам $\left[\mathrm{x}_{\mathrm{k}}-\mathrm{h}, \mathrm{x}_{\mathrm{k}}+\mathrm{h}\right], \mathrm{k}=1, \ldots, \mathrm{n}$, также равны нулю. Тогда

$$
\begin{aligned}
& R\left(H^{\omega_{+} ; \omega_{-}} ; S\right) \geq \frac{1}{2} \int_{0}^{2 \pi} g_{1}(t) d t-\varphi(0, \ldots, 0)-\int_{0}^{2 \pi} g_{2}(t) d t+\varphi(0, \ldots, 0) \mid \geq \\
& \geq \frac{1}{2}\left(\int_{0}^{2 \pi} g_{1}(t) d t-\int_{0}^{2 \pi} g_{2}(t) d t\right) .
\end{aligned}
$$

Оценим снизу интеграл по периоду функции $g_{1}$.

$$
\begin{aligned}
& \int_{0}^{2 \pi} g_{1}(t) d t=\int_{y_{0}}^{y_{0}} g_{1}(t) d t=\sum_{k=1}^{n}\left(\int_{y_{k-1}}^{x_{k}-h} g_{1}(t) d t+\int_{x_{k}-h}^{x_{k}+b} g_{1}(t) d t+\int_{x_{k}+h}^{y_{k}} g_{1}(t) d t\right)= \\
& =\sum_{k=1}^{n}\left(\int_{y_{k-1}}^{x_{k}-h} \bar{g}_{0}\left(t-x_{k}\right) d t+\int_{x_{k}+h}^{y_{k}+\bar{g}_{0}}\left(t-x_{k}\right) d t\right)=\sum_{k=1}^{n}\left(\int_{h}^{x_{k}-y_{k-1}} \int_{0}(-t) d t+\int_{b}^{y_{k}-x_{k}} \bar{g}_{0}(t) d t\right)= \\
& =n\left(\frac{1}{n} \sum_{k=1}^{n} G_{-}\left(x_{k}-y_{k-1}\right)+\frac{1}{n} \sum_{k=1}^{n} G_{+}\left(y_{k}-x_{k}\right)\right),
\end{aligned}
$$

где $G_{ \pm}(x)=\int_{h}^{x} \bar{g}_{0}( \pm t) d t$. Так как $\overline{\mathrm{g}}_{0}(\mathrm{t})$ и $\overline{\mathrm{g}}_{0}(-\mathrm{t})$ - неубывающие на $[\mathrm{h},+\infty)$, то $G_{+}(t)$ и $G_{-}(t)$ - выпууклы вниз на $[h,+\infty)$. Поэтому

$$
\int_{0}^{2 \pi} g_{1}(t) d t \geq n\left(G_{-}\left(\frac{1}{n} \sum_{k=1}^{n}\left(x_{k}-y_{k-1}\right)\right)+G_{+}\left(\frac{1}{n} \sum_{k=1}^{n}\left(y_{k}-x_{k}\right)\right)\right) .
$$

Пусть $\mathrm{p}=\frac{1}{\mathrm{n}} \sum_{\mathrm{k}=1}^{\mathrm{n}}\left(\mathrm{x}_{\mathrm{k}}-\mathrm{y}_{\mathrm{k}-1}\right)$, тогда $\frac{1}{\mathrm{n}} \sum_{\mathrm{k}=1}^{\mathrm{n}}\left(\mathrm{y}_{\mathrm{k}}-\mathrm{x}_{\mathrm{k}}\right)=\frac{2 \pi}{\mathrm{n}}-\mathrm{p}$, и

$$
\int_{0}^{2 \pi} g_{1}(t) d t \geq n\left(G_{-}(p)+G_{+}\left(\frac{2 \pi}{n}-p\right)\right)=n\left(\int_{h}^{p} \bar{g}_{0}(-t) d t+\int_{h}^{\frac{2 \pi}{n} p} \bar{g}_{0}(t) d t\right)
$$

Учитьвая, что $\overrightarrow{\mathrm{g}}_{0}$ на $\{-\mathrm{h}, \mathrm{h}\}$ в среднем равна нулю, иделая замену в первом.интеграле, получим

$\int_{0}^{2 \pi} g_{1}(t) d t \geq n\left(\int_{-p}^{-h} \bar{g}_{0}(t) d t+\int_{-h}^{h} \bar{g}_{0}(t) d t+\int_{h}^{\frac{2 \pi}{n} p} \bar{g}_{0}(t) d t\right)=n \int_{-p}^{\frac{2 \pi}{n} p} \bar{g}_{0}(t) d t \geq n \int_{\mu_{0}}^{\mu_{0}+\frac{2 \pi}{\underline{n}}}(t) d t$

Аналогично доказывается, что 


$$
-\int_{0}^{2 \pi} g_{2}(t) d t \geq n \int_{\mu_{0}}^{\mu_{0}+\frac{2 \pi}{g_{0}}}(t) d t
$$

Tогда, с учетом (8),

$$
R\left(\mathrm{H}^{\omega_{+}+\omega_{-}} ; \mathrm{S}\right) \geq \mathrm{n} \int_{\mu_{0}}^{\mu_{0}+\frac{2 \pi}{\mathrm{n}}}(\mathrm{t}) \mathrm{dt}=\mathrm{n}\left(1-\frac{\mathrm{hn}}{\pi}\right) \int_{0}^{\frac{2 \pi}{\mathrm{n}}} \min \left\{\omega_{+}(\mathrm{t}) \omega_{-}\left(\frac{2 \pi}{\mathrm{n}}-\mathrm{t}\right)\right\} \mathrm{dt} .
$$

Следовательно,

$$
\begin{aligned}
R_{h, a}\left(H^{\omega_{+}+\omega_{-}}\right)=\inf _{S \in D_{h, a}} R\left(H^{\omega_{+} ; \omega_{-} ; S}\right) \geq \\
\geq n\left(1-\frac{h n}{\pi}\right) \int_{0}^{\frac{2 \pi}{n}} \min \left\{\omega_{+}(t) \omega_{-}\left(\frac{2 \pi}{n}-t\right)\right\} d t ;
\end{aligned}
$$

и так как $\mathrm{R}_{\mathrm{h}, \mathrm{n}}\left(\mathrm{H}^{\omega_{*} ; \omega_{-}}\right) \leq \mathrm{R}\left(\mathrm{H}^{\omega_{*} ; \omega_{-}} ; \mathrm{S}^{*}\right)$ и для алгоритма $\mathrm{S}^{*}$ выполнякотся неравенства (4) и (9), то

$$
\mathrm{R}_{\mathrm{h}, \mathrm{n}}\left(\mathrm{H}^{\mathrm{\omega}_{+} \omega_{-}}\right)=\mathrm{R}\left(\mathrm{H}_{+}^{\omega_{+} ; \omega_{-} ; \mathrm{S}^{*}}\right)=\mathrm{n}\left(1-\frac{\mathrm{hn}}{\pi}\right) \int_{0}^{\frac{2 \pi}{\mathrm{n}}} \min \left\{\omega_{+}(\mathrm{t}) \omega_{-}\left(\frac{2 \pi}{\mathrm{n}}-\mathrm{t}\right)\right\} \mathrm{dt} .
$$

Таким образом, алгоритм $\mathrm{S}^{*}$ является оттимальным на классе $\mathrm{H}^{\omega_{+} ; \omega_{-}}$среди апгаиттіов множества $D_{h, n}$.

Теорема доказана.

Автор выражает благодарность Бабенко В.Ф. за ценные советы при проведении исследования.

\section{Библиографические ссылки}

1: Бабенко В.Ф. Об одной задаче, оптимизации прнближкенного интегрирования // Исслед. по совр. пробл. суммирования и приближения функций и их приложениям. Джегропетровск, 1984. С.3-13.

2. Бабенко В.Ф., Поляков О.В. Приблюжения в среднем некоторьх классов непрерывных периодических фунхихй // Изв. вузов. 1995. № 2. С.13-21.

3. Корнейчук Н.П. Экстремаљьные задачи теории приблкженкя. М., 1979.

4. Кузьмина А.А. Ннтервальные квадратурные формулы с. кратными узловыми интервалами // Изв. вузов.. 1980. № 7. С.39-44.

5. Нккольский С.М. Квадратурные формулы. М., 1979.

6. ÚІарипов Р.Н. Наялучпшие интервальные квадратурные формулы для классов Литшица // Конструктив. теория функций и функщион. анализ. Казань, 1983. Вып. 4. С.124-132.

7. Omladic M., Pahor S., Suhadolc A. On a new type of quadrature formulas // Numer. Math.. 1976. Bd. 25, № 4. P.421-426.

8. Pittnauer Fr., Reimer M. Interpolation mit Intervallfunktionalen // Math. Z. 1976. Bd.146, № 1. P.7-15. 